\title{
Measuring longitudinal amplitudes for electroproduction of pseudoscalar mesons using recoil polarization in parallel kinematics
}

\author{
James J. Kelly \\ Department of Physics, University of Maryland, College Park, MD 20742
}

(November 12, 2017)

\begin{abstract}
We propose a new method for measuring longitudinal amplitudes for electroproduction of pseudoscalar mesons that exploits a symmetry relation for polarization observables in parallel kinematics. This polarization technique does not require variation of electron scattering kinematics and avoids the major sources of systematic errors in Rosenbluth separation.
\end{abstract}

Transition form factors for electroexcitation of nucleon resonances provide important tests of QCD-inspired models baryon structure. However, it is often very difficult to separate unpolarized longitudinal response functions from the dominant transverse response functions using the traditional Rosenbluth method without substantial systematic errors arising from the strong dependence of both acceptances and cross sections upon electron-scattering kinematics. Arnold, Carlson, and Gross [1] demonstrated that the ratio between electric and magnetic nucleon elastic form factors can be measured using either recoil or target polarization; such techniques are now becoming standard for elastic scattering.

In this Brief Report we demonstrate that polarization observables for electroproduction of pseudoscalar mesons in parallel kinematics can be used to separate longitudinal and transverse amplitudes without need of Rosenbluth separation. When the nucleon momentum and spin are both parallel to the momentum transfer, conditions sometimes described as superparallel kinematics [2], the polarized and unpolarized transverse response functions become identical and the recoil polarization or the target polarization asymmetry can be used to determine the ratio between longitudinal and transverse cross sections $[3$ 月. Some of the implications of this symmetry have been considered for nucleon knockout reactions upon spin-0 targets which leave the residual nucleus with spin- $\frac{1}{2}$ [6] and for electron scattering by a polarized spin- $\frac{1}{2}$ target [7]. Raskin and Donnelly [8] also mention this symmetry for pion electroproduction. Using the nonstandard multipole expansion of Raskin and Donnelly and assuming dominance of the $M_{1+}$ amplitude for the $N \rightarrow \Delta$ transition, Schmieden [9] discussed the sensitivity of polarization observables for pion electroproduction to quadrupole amplitudes, but the symmetry is more general and it is not necessary to assume that a single resonance dominates. The complete tables of response functions expressed in terms of helicity amplitudes for pseudoscalar meson production which can be found in Refs. 10,11] implicitly contain the results below also. Nevertheless, here we present some of the practical aspects of exploiting this symmetry because the simplicity and utility of the polarization method for superparallel kinematics does not appear to be widely known or appreciated.

The reaction amplitudes for any $A\left(e, e^{\prime} N\right) B$ process where $A$ has spin- $\frac{1}{2}$ and $B$ spin-0 that is governed by the one-photon exchange mechanism can be expressed in terms of helicity amplitudes of the form

$$
H_{\lambda_{f} \lambda_{i} \lambda_{\gamma}}\left(Q^{2}, W, \theta, \phi\right)=\left\langle\lambda_{f}\left|\mathcal{F}_{\mu} \varepsilon^{\mu}\right| \lambda_{i}, \lambda_{\gamma}\right\rangle
$$

where $\lambda_{i}$ and $\lambda_{f}$ are the initial and final helicities of the nucleon, $\lambda_{\gamma}$ is the helicity of the virtual photon, $\mathcal{F}^{\mu}$ is an appropriately normalized transition current operator, and $\varepsilon^{\mu}$ is the virtual-photon polarization vector. The invariant mass of the meson-nucleon system is given by $W$, while $Q^{2}=\mathbf{q}^{2}-\omega^{2}$ is the virtuality of a spacelike photon. The pion direction relative to the momentum transfer $\mathbf{q}$ and electron-scattering plane is given by polar and azimuthal angles $\theta$ and $\phi$. We label a nucleon recoil momentum that is along $\mathbf{q}$, such that $\theta=\pi$, as parallel or a nucleon momentum in the opposite direction as antiparallel, and assign $\phi=0$ to both. Phase conventions for helicity states follow the conventions of Jacob and Wick [12].

The present derivation assumes that the reaction is mediated by one-photon exchange and conserves parity, but makes no other assumptions about the details of the transition amplitudes. Since parity conservation 12 requires $\left|H_{-\lambda_{f}-\lambda_{i}-\lambda_{\gamma}}\right|=\left|H_{\lambda_{f} \lambda_{i} \lambda_{\gamma}}\right|$, it is sufficient to consider six independent amplitudes $H_{i}$ for $\left(\lambda_{f}, \lambda_{i}, \lambda_{\gamma}\right)$ chosen as $\left(-\frac{1}{2},-\frac{1}{2}, 1\right),\left(-\frac{1}{2}, \frac{1}{2}, 1\right),\left(\frac{1}{2},-\frac{1}{2}, 1\right),\left(\frac{1}{2}, \frac{1}{2}, 1\right),\left(\frac{1}{2}, \frac{1}{2}, 0\right)$, and $\left(\frac{1}{2},-\frac{1}{2}, 0\right)$ and numbered sequentially 13.14. Due to the absence of orbital angular momentum in the initial state or spin in the undetected recoil particle $(B)$, the angular momentum projected onto the virtual photon direction reduces to $J_{z}=\lambda_{\gamma}-\lambda_{i}= \pm \lambda_{f}$ for parallel or antiparallel kinematics, where the upper sign applies to parallel and the lower to antiparallel kinematics. Hence, only $H_{4}$ and $H_{6}$ contribute to parallel or $H_{2}$ and $H_{5}$ to antiparallel kinematics. It is convenient to define $T_{+}=H_{4}$ and $L_{+}=H_{6}$ as the transverse and longitudinal amplitudes relevant to parallel kinematics and $T_{-}=-H_{2}$ and $L_{-}=H_{5}$ as the corresponding amplitudes for antiparallel kinematics. These amplitudes are related to the usual CGLN [15, 16] coefficients by 


$$
\begin{aligned}
& T_{ \pm}=\sqrt{2}\left(\mathcal{F}_{1} \pm \mathcal{F}_{2}\right) \\
& L_{ \pm}=\frac{Q}{\omega}\left(\mathcal{F}_{5}^{\prime} \mp \mathcal{F}_{6}^{\prime}\right)
\end{aligned}
$$

where

$$
\begin{aligned}
i \mathcal{F}^{0} & =\frac{q}{\omega}\left(\mathcal{F}_{5}^{\prime} \vec{\sigma} \cdot \hat{q}+\mathcal{F}_{6}^{\prime} \vec{\sigma} \cdot \hat{p}\right) \\
i \overrightarrow{\mathcal{F}} & =\mathcal{F}_{1} \vec{\sigma}-i \mathcal{F}_{2} \vec{\sigma} \cdot \hat{p} \vec{\sigma} \times \hat{q}+\mathcal{F}_{3} \hat{p} \vec{\sigma} \cdot \hat{q}+\mathcal{F}_{4} \hat{p} \vec{\sigma} \cdot \hat{p}+\mathcal{F}_{5} \hat{q} \vec{\sigma} \cdot \hat{q}+\mathcal{F}_{6} \hat{q} \vec{\sigma} \cdot \hat{p}
\end{aligned}
$$

and

$$
\begin{aligned}
& \mathcal{F}_{5}^{\prime}=\mathcal{F}_{5}+\mathcal{F}_{3} \hat{p} \cdot \hat{q}+\mathcal{F}_{1} \\
& \mathcal{F}_{6}^{\prime}=\mathcal{F}_{6}+\mathcal{F}_{4} \hat{p} \cdot \hat{q} .
\end{aligned}
$$

Using the standard multipole expansion of CGLN amplitudes introduced by Dennery [16], the amplitudes for antiparallel kinematics become

$$
\begin{aligned}
T_{-} & =\sqrt{\frac{1}{2}} \sum_{\ell}\left[(\ell+1)(\ell+2) E_{\ell+}+\ell(\ell-1) E_{\ell-}+\ell(\ell+1)\left(M_{\ell+}-M_{\ell-}\right)\right] \\
L_{-} & =\frac{Q}{q} \sum_{\ell}\left[(\ell+1)^{2} S_{\ell+}+\ell^{2} S_{\ell-}\right]
\end{aligned}
$$

while the summands for parallel kinematics require an extra factor of $(-)^{\ell}$. Note that because Raskin and Donnelly [8] confused $\theta_{\pi}$ with $\theta_{N}$, the multipole amplitudes used by Schmieden [9] should be multiplied by $(-)^{\ell}$. Furthermore, Raskin and Donnelly give the opposite sign for $E_{\ell-}$.

The differential cross section for the meson electroproduction reaction $p\left(\vec{e}, e^{\prime} \vec{N}\right) x$ can be expressed in the form

$$
\frac{d^{5} \sigma}{d k_{f} d \Omega_{e} d \Omega_{N}}=\Gamma_{\gamma} \sigma_{v}
$$

where $\sigma_{v}$ is the center of mass cross section for the virtual photoproduction reaction $\gamma_{v}+N \rightarrow x+N$ and

$$
\Gamma_{\gamma}=\frac{\alpha}{2 \pi^{2}} \frac{k_{f}}{k_{i}} \frac{k_{\gamma}}{Q^{2}} \frac{1}{1-\epsilon}
$$

is the virtual photon flux for initial (final) electon momenta $k_{i}\left(k_{f}\right)$. Here $\epsilon=\left(1+2 \frac{\mathbf{q}^{2}}{Q^{2}} \tan ^{2} \frac{\theta_{e}}{2}\right)^{-1}$ is the transverse polarization of the virtual photon, $\theta_{e}$ is the electron scattering angle, and $k_{\gamma}=\left(W^{2}-m_{p}^{2}\right) / 2 m_{p}$ is the laboratory energy a real photon would need to excite the same transition. The spin dependence of the virtual photoproduction cross section for an unpolarized target can be expressed in the form

$$
\sigma_{v}=\bar{\sigma}\left[1+\boldsymbol{P} \cdot \boldsymbol{\sigma}+h\left(A+\boldsymbol{P}^{\prime} \cdot \boldsymbol{\sigma}\right)\right]
$$

where $\bar{\sigma}$ is the unpolarized differential cross section, $A$ is the beam analyzing power, $\boldsymbol{P}$ is the induced or helicityindependent recoil polarization, $\boldsymbol{P}^{\prime}$ is the polarization transfer or helicity-dependent recoil polarization, and $h$ is the beam helicity. Thus, the net polarization of the recoil nucleon is $\boldsymbol{\Pi}=\boldsymbol{P}+h \boldsymbol{P}^{\prime}$. A similar expression applies when the target is polarized and the recoil polarization is unobserved. We omit observables requiring both target and recoil polarization because they provide no new information for parallel kinematics and are so difficult to measure as to be of no practical interest. For parallel kinematics it is simplest to refer polarizations to a basis in which $\hat{\boldsymbol{z}}=\hat{\boldsymbol{q}}$ is in the photon direction, $\hat{\boldsymbol{y}}=\hat{\boldsymbol{k}}_{i} \times \hat{\boldsymbol{k}}_{f}$ is normal to the electron scattering plane, and $\hat{\boldsymbol{x}}=\hat{\boldsymbol{y}} \times \hat{\boldsymbol{z}}$ is transverse.

Recoil polarization observables can now be expressed in the form

$$
\begin{aligned}
\bar{\sigma} & =\sigma_{T}+\epsilon \sigma_{L}=\mathcal{K}\left(\frac{1}{2}|T|^{2}+\epsilon|L|^{2}\right) \\
\Pi_{x} \bar{\sigma} & =h \mathcal{K} \sqrt{\epsilon(1-\epsilon)} \operatorname{Re}\left(T L^{*}\right) \\
\Pi_{y} \bar{\sigma} & =-\mathcal{K} \sqrt{\epsilon(1+\epsilon)} \operatorname{Im}\left(T L^{*}\right) \\
\Pi_{z} \bar{\sigma} & =h \mathcal{K} \sqrt{1-\epsilon^{2}} \frac{1}{2}|T|^{2}
\end{aligned}
$$


where $\mathcal{K}=p W / k_{\gamma} m_{p}$ is a kinematic factor and $p$ is the final center of mass momentum. We have left the \pm subscripts on observables and amplitudes implicit in the interests of brevity. In the chosen basis target and recoil polarization observables for parallel kinematics differ only in sign. Thus, using either recoil or target polarization, there are five observables that depend upon just four response functions (bilinear amplitude products). Therefore, there exists a relationship between polarization and cross section for parallel kinematics that provides an alternative method for separating the longitudinal and transverse cross sections.

We define

$$
\mathcal{R}_{ \pm}=\frac{\sigma_{L \pm}}{\sigma_{T \pm}}=2 \frac{\left|L_{ \pm}\right|^{2}}{\left|T_{ \pm}\right|^{2}}
$$

as the ratio between longitudinal and transverse cross sections for parallel kinematics. The traditional Rosenbluth separation method relies on the variation of cross section with $\epsilon$, but this method requires measurements for two or more electron scattering kinematics with quite different acceptances. When the longitudinal contribution is small, the systematic errors due to acceptances and kinematic variables can become prohibitively large. Alternatively, for parallel kinematics it is possible to exploit the relationship between the longitudinal component of recoil (or target) polarization and the transverse contribution to the differential cross section to obtain

$$
\mathcal{R}=\frac{h \sqrt{1-\epsilon^{2}}-\Pi_{z}}{\epsilon \Pi_{z}}
$$

with fixed electron scattering kinematics without Rosenbluth separation; in fact, for the ratio one does not even need to normalize the cross section. Therefore, this polarization technique avoids the major sources of systematic error that afflict the Rosenbluth method.

Assuming that $\epsilon$ is known accurately, the uncertainty in $\mathcal{R}$ is related to the polarization uncertainty $\delta \Pi_{z}$ by

$$
\frac{\delta \mathcal{R}}{\delta \Pi_{z}}=\frac{|h| \sqrt{1-\epsilon^{2}}}{\epsilon \Pi_{z}^{2}}=\frac{(1+\mathcal{R} \epsilon)^{2}}{|h| \epsilon \sqrt{1-\epsilon^{2}}} .
$$

If $\mathcal{R}$ is small and if the minimum attainable value of $\delta \Pi_{z}$ is governed by systematic errors, then the optimum kinematics for measurement of $\mathcal{R}$ are realized when $\epsilon=1 / \sqrt{2}$. Alternatively, when statistical uncertainties dominate $\delta \Pi_{z}$, it becomes advantageous to employ the largest practical $\epsilon$ because the virtual-photon flux is proportional to $(1-\epsilon)^{-1}$; for given $W$ and $Q^{2}$ this implies that higher beam energies are more favorable.

These relationships can also be used to establish a bound

$$
\left|\Pi_{z}\right| \leq|h| \sqrt{1-\epsilon^{2}}
$$

upon the longitudinal polarization, where the limiting value is realized for a purely transverse electroproduction amplitude. Recognizing that in parallel kinematics the transverse amplitudes flip nucleon spin while the longitudinal amplitudes do not, one finds that reduction of the longitudinal polarization from its maximal value would be indicative of a measurable spin nonflip amplitude. Furthermore, a nonvanishing normal component of polarization is indicative of a phase difference between longitudinal and transverse amplitudes. The magnitudes of the longitudinal and transverse helicity amplitudes and the relative phase between them can be determined from

$$
\begin{aligned}
L & =r T e^{i \delta} \\
|T|^{2} & =\frac{2 \bar{\sigma} \Pi_{z}}{h \mathcal{K} \sqrt{1-\epsilon^{2}}} \\
r^{2} & =\mathcal{R} / 2=\frac{h \sqrt{1-\epsilon^{2}}-\Pi_{z}}{2 \epsilon \Pi_{z}} \\
\tan \delta & =\sqrt{\frac{1-\epsilon}{1+\epsilon}} \frac{\Pi_{y}}{\Pi_{x}} h .
\end{aligned}
$$

Finally, under some conditions these quantities provide useful constraints upon multipole amplitudes. For example, if we limit the expansions to $s$ - and $p$-waves and include only contributions involving the dominant $M_{1+}$ amplitude for pion electroproduction near the $P_{33}(1232)$ resonance, we find

$$
\operatorname{Re}\left(S_{1-}+4 S_{1+} \mp S_{0+}\right) M_{1+}^{*} \approx \frac{\Pi_{x \pm} \bar{\sigma}_{ \pm}}{h \sqrt{2} \frac{Q}{q} \mathcal{K} \sqrt{\epsilon-\epsilon^{2}}} .
$$


Thus, by comparing parallel versus antiparallel kinematics one can separate the combinations $\operatorname{Re} S_{0+} M_{1+}^{*}$ and $\operatorname{Re}\left(S_{1-}+4 S_{1+}\right) M_{1+}^{*}$. Most attempts to measure the $S_{1+}$ amplitude for pion electroproduction, which is sensitive to quadrupole deformation of the nucleon and $\Delta$ wave functions, have relied upon the $R_{L T}$ response function obtained from the left-right asymmetry of the unpolarized cross section. However, Mertz et al. [17] have shown that current models fail to reproduce the $W$ dependence of the cross section asymmetry, which casts doubt upon the reliability of fitted $S_{1+}$ resonance amplitudes. It is widely believed that the $S_{0+}$ contribution may be responsible for these difficulties, but it should be possible to measure this amplitude with relatively little model dependence using recoil polarization for parallel kinematics. Furthermore, although one cannot separate $S_{1+}$ from $S_{1-}$ without more comprehensive data, the $S_{1-}$ contribution is expected to be quite small and to display a distinctly different dependence upon $W$. Therefore, recoil polarization for parallel versus antiparallel kinematics offers an independent method for measuring $S_{1+}$ also.

In summary, we have proposed a polarization method for measuring the ratio between longitudinal and transverse cross sections for electroproduction of pseudoscalar mesons in parallel kinematics that employs fixed electron kinematics and avoids Rosenbluth separation. We have also developed the relationships needed to extract the magnitudes and relative phase of the corresponding helicity amplitudes. It is important to recognize that this method does not depend upon dominance of any particular resonance and applies equally well to the resonant and nonresonant contributions. However, it need not apply to more complicated background processes such as $p\left(e, e^{\prime} N\right) \pi \pi$. Fortunately, for many interesting experiments, such as $\gamma_{v} N \rightarrow P_{33}(1232) \rightarrow N \pi$ or $\gamma_{v} N \rightarrow S_{11}(1535) \rightarrow N \eta$, those background contributions should vary slowly with missing mass and can be subtracted from the single-meson peak in the missing mass distribution. Nor can this method be used to obtain full angular distributions for longitudinal and transverse response functions. Nevertheless, the ability to separate longitudinal and transverse amplitudes in parallel and/or antiparallel kinematics using recoil polarization measurements without Rosenbluth separation can be very helpful in testing models of baryon structure and is a useful supplement to the traditional cross section method.

[1] R. G. Arnold, C. E. Carlson, and F. Gross, Phys. Rev. C 23, 363 (1981).

[2] S. Boffi, C. Giusti, and F. D. Pacati, Phys. Rep. 226, 1 (1993).

[3] J. J. Kelly, Adv. Nucl. Phys. 23, 75 (1996).

[4] J. J. Kelly et al., Recoil Polarization in $\eta$ Electroproduction, TJNAF Proposal 96-001, 1996.

[5] J. J. Kelly et al., The $p\left(\vec{e}, e^{\prime} \vec{N}\right) \pi$ Reaction with Harp, TJNAF Proposal 93-013, 1993.

[6] S. Boffi, C. Giusti, and F. D. Pacati, Nucl. Phys. A476, 617 (1988).

[7] C. Giusti and F. D. Pacati, Nucl. Phys. A504, 685 (1989).

[8] A. S. Raskin and T. W. Donnelly, Ann. Phys. (N.Y.) 191, 78 (1989).

[9] H. Schmieden, Eur. Phys. J. A1, 427 (1998).

[10] G. Knöchlein, D. Drechsel, and L. Tiator, Zeit. Phys. A 352, 327 (1995).

[11] D. Drechsel and L. Tiator, J. Phys. G 18, 449 (1992).

[12] M. Jacob and G. C. Wick, Ann. Phys. (N.Y.) 7, 404 (1959).

[13] H. F. Jones, Il Nuovo Cimento 40A, 1018 (1965).

[14] R. L. Walker, Phys. Rev. 182, 1729 (1969).

[15] G. F. Chew, M. L. Goldberger, F. E. Low, and Y. Nambu, Phys. Rev. 106, 1345 (1957).

[16] P. Dennery, Phys. Rev. 124, 2000 (1961).

[17] C. Mertz et al., submitted to PRL (unpublished). 